\title{
Research on Evolutionary Design Method of BIM Technology in Highway Reconstruction and Expansion Project
}

\author{
Zhou Leisheng ${ }^{1}$, Chen Yinghao $^{2}$, Xia Jianping ${ }^{1}$ Liu cheng $^{2}$ and Yang guangliang ${ }^{1}$ \\ ${ }^{1}$ Qilu Transportation Development Group Co., Ltd, Jinan, Shandong, 250001, China \\ ${ }^{2}$ Institute of Highway Science, Ministry of Transport, Beijing, 100088, China
}

\begin{abstract}
BIM technology is widely used in expressway Construction projects. In the process of transforming two-dimensional design into three-dimensional design, there are still many problems that need to be solved urgently. Based on the Beijing-Shanghai highway reconstruction and expansion project, this paper proposes an evolutionary design method. Based on the application standard of project model technology, the method uses BIM model software, based on skeleton lines and templates, using EKL programming language to realize the structure size from coarse to fine, information from summary to detail, and progressive design method for component granularity from bottom to high. At the same time, a three-dimensional design method for automatically generating road centerlines is proposed. The results show that the evolutionary design method is in line with the current project habits, matching the existing design concepts, and can efficiently complete the design work at different stages, effectively avoiding a lot of repetitive work.
\end{abstract}

\section{Introduction}

BIM technology is widely used in the highway engineering, especially in large highway, bridges and tunnels. BIM also plays a significant role in the survey, design, construction and management in infrastructure projects, improving the overall design capabilities and fine management level ${ }^{[1]}$. However, many problems remain to be solved urgently from $2 \mathrm{D}$ design to $3 \mathrm{D}$ design ${ }^{[2]}$. For example, BIM-based design usually increases work hour, as most existing highway projects are based on 2D design. Besides, how to build BIM model finely, fast and efficiently is yet to be solved. Many have used BIM technology to study the design and application of highway projects. Heap ${ }^{[3]}$ conducted a comparative study of BIM in infrastructure projects and building projects. Blaine ${ }^{[4]}$ studied the differences in the application of BIM technology in two Bridges. Song Fuchun ${ }^{[5]}$ studied the application of BIM technology in the design of cablestayed bridges. Dong Jun ${ }^{[6]}$ studied the application of BIM technology in highway construction.

Most existing literature focuses on newly-built projects. However, the fact that BIM model is iteratively updated during the lifetime of the projects is rarely considered. This paper proposes an evolutionary design method of BIM technology. The proposed design method in applied in Beijing-Shanghai highway reconstruction and expansion projects; a parametric, automatic and iterable BIM modeling solution to general highway reconstruction and expansion projects is also concluded.

\section{Engineering situation}

The Beijing-Shanghai highway from Xintai Hub to Linyi section (hereinafter called as the Xintai-Linyi highway) was completed and opened to traffic in 1999. The original design of the Xintai-Linyi highway is a two-way four-lane expressway with a width of 28.0 meters and a design speed of $120 \mathrm{~km} / \mathrm{h}$. With the rapid development of China's economy and society, the expressway transportation capacity of the pre-construction is tight and the service level is seriously degraded. Therefore, it is necessary to renovate and expand the Xintai-Linyi section of the Beijing-Shanghai Expressway. 


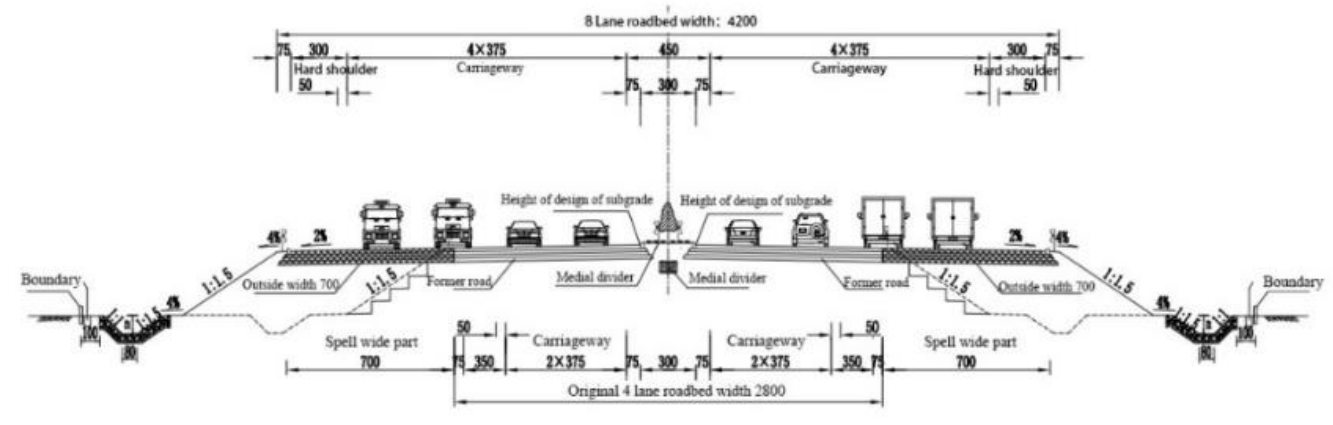

Figure 1. Cross-sectional view of the double-sided widened roadbed of the main line of Beijing-Shanghai highway reconstruction and expansion

The Beijing-Shanghai renovation and expansion project adopts the highway standard of fully enclosed and fully interchanged. Adopting a two-way eight-lane standard, the roadbed is $42 \mathrm{~m}$ wide (as shown in Figure 1). Among them, there are 4 special bridges, 36 bridges, 37 middle bridges, 363 small bridges and passages, 74 flyovers, 1 tunnel, 21 interchanges and 7 service areas. Considering the importance of the project and the complexity of the expansion and expansion, it is necessary to use BIM technology to refine it.

\section{Model technology application standard}

\subsection{Necessity}

In order to unify the application requirements of the building information model of the project, standardize and guide the application of highway engineering information model technology throughout the life cycle, improve the quality of highway engineering, and implement the concept of life-cycle management, it is necessary to establish project model technology application standards.

BIM technology is based on models, while models should be based on standards ${ }^{[7]}$. The development of BIM project work usually involves different professions and collaboration between different teams. At the beginning of the project, the unified model standard can ensure that the collaborative work can be carried out smoothly and effectively; in the process of project development, the unified model standard can guarantee the unification of various model forms, formats and types; after the project is completed, it will be unified after operation and maintenance. The model standard guarantees the complete application of BIM throughout the life of the project, making it easy to call and manage.

\subsection{Model standard content}

The technical standards for the Beijing-Shanghai reform and expansion model mainly include two modules: modeling standards and model delivery standards. For the evolutionary design concept proposed in this paper, the standardization and rigor of the modeling standard is the prerequisite for ensuring high quality design. The Beijing-
Shanghai project modeling standard mainly includes the following parts:

(1) Model spatial positioning;

(2) Project structure tree division;

(3) Product structure level relationship;

(4) Template classification and management;

(5) product color matching and naming rules;

(6) attribute expansion and coding rules;

(7) Model review.

The BIM model data storage of the Beijing-Shanghai reform and expansion project is based on the IFC $4 \times 1$ standard established by the building SMART organization and is expanded according to the needs of the project. Establish project-specific exclusive product IfcRoad, expand its properties and materials as needed, and its Express expression is as follows:

ENTITY IfcRoad

SUBTYPE OF (IfcBuiltFacility);

END ENTITY;

USERDEFINED: user defined;// Type enumeration definition for the road entity

NOTDEFINED: Undefined

EXPRESS:

TYPE IfcRoadTypeEnum = ENUMERATION OF

(USERDEFINED,

NOTDEFINED);

END TYPE;

\section{Evolutionary design method}

The evolutionary design method proposed in this paper is applicable to the design and application of BIM model from preliminary design to construction drawing design to construction deepening. Its essence is to use extended attribute interface, skeleton line thinking, parameterized driving, script batch instantiation and iteration. Update the design, implement the same skeletal model, and gradually deepen the design process at different stages. In the design process, the model is gradually deepened and improved, and the purpose of the design process and the fineness of the model is closely combined.

\subsection{Attribute expansion}

Relying on the above model technical standards, using CATIA as a modeling software, build project-specific products, and expand the IFC properties according to the 
Express language. The extended attribute information includes GKBIM WBS, GKBIM DESCRIPTION, GKBIM_MATERIAL, and $\overline{\text { GKBIM_COLOR, }}$ corresponding to the code, Chinese description, component material information, and color (hexadecimal code). After the attribute is extended to the attribute bar, the EKL script language and rules are used to realize the linkage between the parameter and the attribute column. The product is instantiated in batches, and the attributes are also entered in batches according to different contents. The overall idea of attribute expansion is shown in Figure 2 .

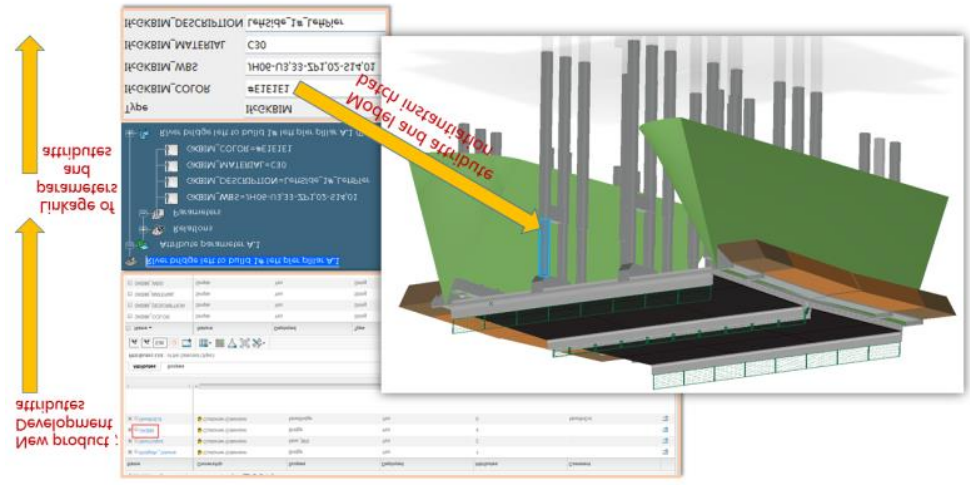

Figure 2. Schematic diagram of model attribute expansion and instantiation

\subsection{Parameterized skeleton}

For high-speed projects, the skeleton line refers to the road center line reflecting the plane position and straightness of the road, and the control position of each bridge pier and the axis system at the center of the culvert.

The complete road module is not built in CATIA. This paper uses EKL script language to realize the automatic generation of road centerline, which effectively improves the modeling efficiency of skeleton line. The flow chart is shown in Figure 3. The main steps are: arranging the flat curve element and the vertical curve element table $\rightarrow$ establishing the resource table $\rightarrow$ writing the script in EKL language, reading the flat curve element table to realize the establishment of the flat curve $\rightarrow$ writing the script in EKL language, read the vertical curve element table to achieve the establishment of a flat curve $\rightarrow$ use the EKL language and built-in expansion folding command to generate the road center line.

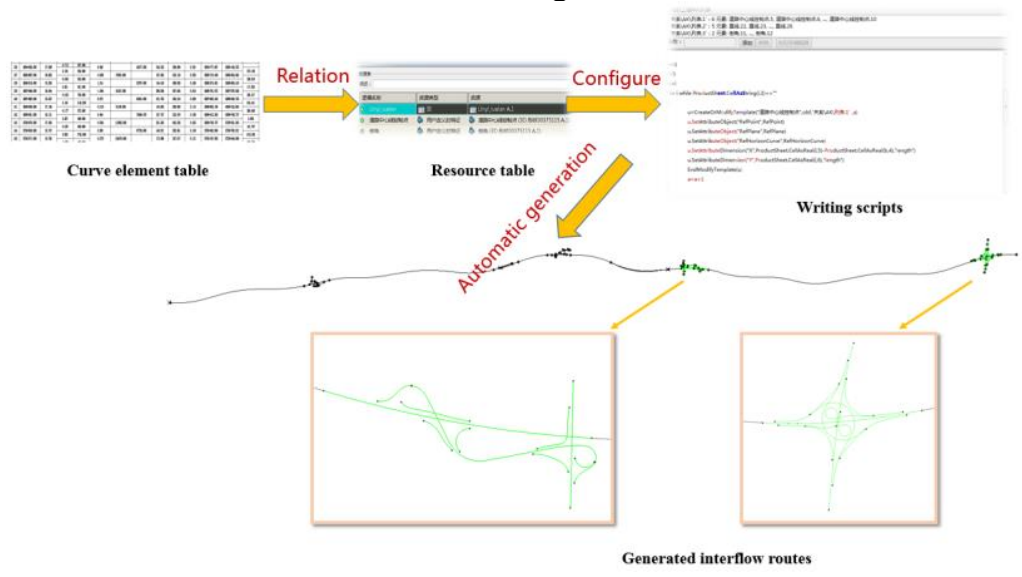

Figure 3. Schematic diagram of the road centerline automated modeling process

\subsection{Parametric design}

Parametric design can effectively improve design efficiency ${ }^{[8]}$, allowing designers to focus more on the design itself. For the reconstruction and expansion of highway projects, its parametric design mainly includes skeleton parameterization, component parameterization and attribute parameterization. Explain as follows:

(1) Skeleton parameterization

The skeleton is a positioning element that supports the formation of points, lines, and surfaces of the entire model. When the skeleton is completed, the microscopic structural changes and positional changes can be achieved by modifying the skeleton to achieve the desired effect. For example, in the design stage, the elevation of a design point of the route needs to be adjusted upward by $2 \mathrm{~m}$ according to the situation of the road. Only the control parameters of the corresponding position of the skeleton need to be adjusted, and the entire road model will be driven, as shown in Figure 4. 


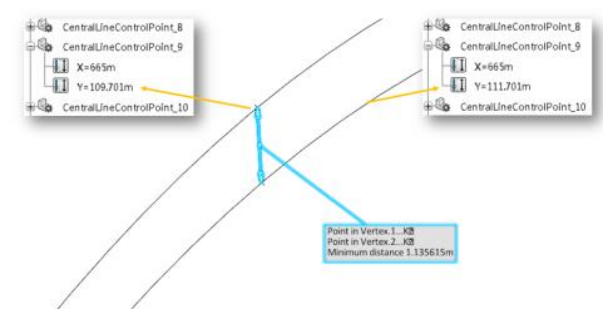

Figure 4. Skeleton parameter drive schematic

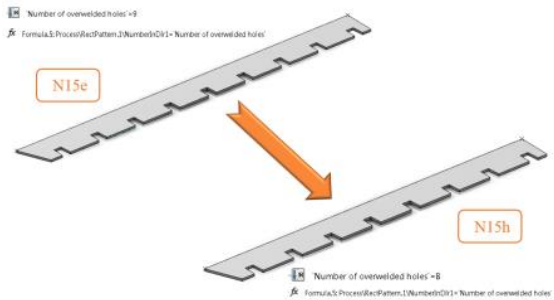

Figure 5. Schematic diagram of component parameterization drive

(2) Component parameterization

By repeating the parameters of a single component, repeated modeling of similar components is avoided, and the model can be applied as a template to the next project. In other words, the long-term significance of component parameterization lies in the accumulation of material library, and the accumulation of long-term material library can make similar projects more efficient to complete the design work. Taking the engineering steel structure stiffening plate as an example, the transverse stiffening plates N15e and N15h are of the same type and different lengths, and the number of over-welded holes is changed from 9 to 8 . For such similar components, only a single template is needed, wherein the length is adaptively adjusted according to the number and spacing of the overwelded holes, and the number of over-welded holes is related by parameters, as shown in Figure 5, modifying the number of over-welded holes in the N15e stiffener template to obtain the N15h stiffener.

(3) attribute parameterization

Using the rule to automatically read the parameters to modify the attribute content is attribute parameterization. With a custom parameterized language, you can read the attribute table and attach it to the model according to established rules during batch instantiation.

\subsection{Batch instantiation}

In the highway reconstruction and expansion project, the bridges and roads are divided into existing and new construction. Firstly, multiple templates are batched and instantiated for existing projects. The EKL language is used to automatically read templates and parameter tables to realize different template calls and instantiation. After completing the existing roads and bridges, use existing projects as reference and input conditions, and use the same method to quickly generate new templates in batches, as shown in Figure 6.

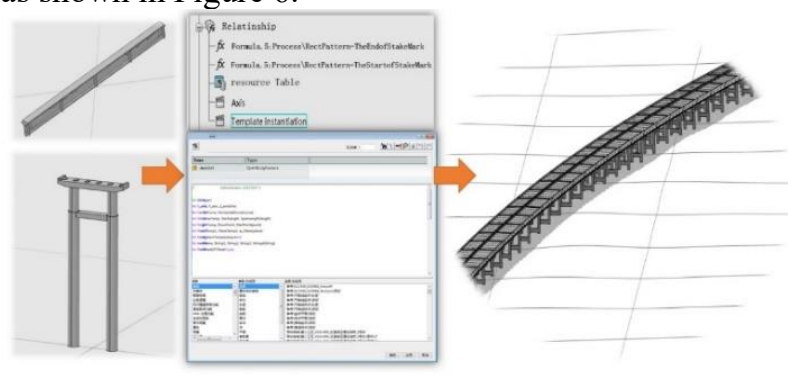

Figure 6. Schematic diagram of the instantiation of new and existing structural bridges

\subsection{Evolutionary iterative design}

The evolutionary design is to gradually improve the existing design ideas, the structure size from rough to precise, the information from the main to the detailed, the component granularity from bottom to high progressive design method.

In the initial stage of business development, the purpose is to determine the position selection and crossline plan. What is needed is not a perfect design model, but a preliminary design model that can express the design idea. At this time, the accuracy of the skeleton line should be considered. For the structure, it can be approximated to ensure that the beam and span arrangement are accurate.

Entering the construction drawing design period, the purpose is to establish a perfect design model, directly refer to the skeleton line of the preliminary design stage, and iteratively upgrade the model on the line. For models that are too extensive, use Template directly for in-place replacement, which is to replace low-level models with high-level models. This is also the conventional method. For models that can be further edited, iteratively update the design, use the existing preliminary design model as a reference, complete the detailed model instantiation, and finally upgrade the preliminary design model, from the part level to the component level. For the steel structure, in order to avoid the cumbersome cross-product operation and volume during the design process, the parts can be built under the same component, and finally the part-tocomponent lifting operation is unified, and the attribute information is given.

In the deepening stage of construction, such as roadbed, side ditch, slope protection, etc., it is necessary to divide the construction sections according to the construction requirements. At this time, the EKL language can be used to divide the batch segments and refine the model granularity.

In the model delivery section, it is necessary to supplement the model. The EKL language can also be used to drive the attributes by parameters to realize the function of increasing or decreasing the attributes of the existing models. 


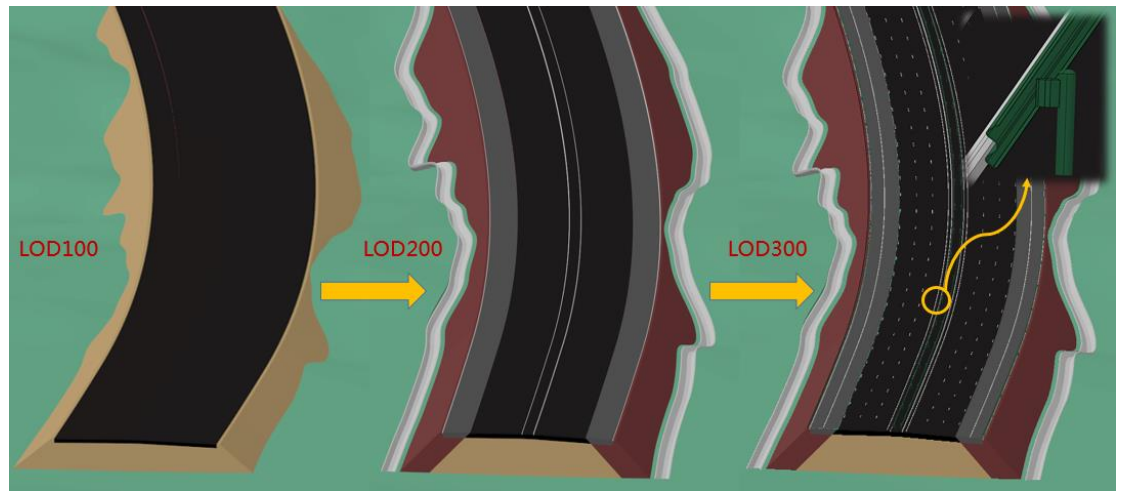

Figure 7. Schematic diagram of the evolutionary design road delivery model

Through the above-mentioned evolutionary design process, with the deepening of design and engineering, the delivery model of the Beijing-Shanghai reconstruction and expansion project was gradually obtained, and the evolution-designed road delivery model was finally obtained as shown in Figure 7.

\section{Conclusion}

(1) This paper establishes the technical standard of BIM model for highway reconstruction and expansion project, and defines project-level products according to project characteristics, and expands its attributes and materials as needed.

(2) This paper uses CATIA modeling software to study an automated route modeling method, which can realize the automatic generation of road centerline.

(3) This paper proposes an advanced evolutionary design method, which is based on skeleton lines and templates, using EKL language for batch instantiation, replacing models according to requirements or performing lifting and lowering operations on models, and completing the refinement of models step by step, effectively improve the efficiency of modeling and avoid repetitive work.

\section{References}

1. Du B.Y., Yan X.Y., He T., et al. (2017) BIM technology architecture and application of expressway industry chain. J. China highway, 11: 134-137.

2. Liu H.M., Zhou Q. (2015) Research and Prospect of BIM Application Status. J. Shandong Industrial Technology, 253: 292.

3. Heap, Y.C., Robert, L., Wang, J., et al. (2016) American Society of Civil Engineers. J. Sci. Commun., 32: 1-13.

4. Blaine, F., Caroline, M.C. (2015) American Society of Civil Engineers. J. Sci. Commun., 20: 1-8.

5. Song F.C., Chen C., Zhang X., et al. (2016) Application of BIM Technology in Long Span Cablestayed Bridge Design. J. Journal of Shenyang Jianzhu University (Natural Science ), 32: 115-123.

6. Dong J., Wang Z.H. (2017) Application of BIM Technology in Expressway Engineering Construction.
J. Highway Engineering, 42: 1-3, 20.

7. Chen Y. H. (2018) Study on the Integrate of Large Cassion Foundation's Design and Construction based on the Application of BIM Technology. D. Chang'an University, Xi'an, China.

8. Hua X.R. (2017) Study on the Application of BIM Technology in the Safety Management in the Construction of Expressway Overpass. J. Highway Engineering, 42: 147-151. 